DFUB $98-19$

Bologna, November 1998

\title{
FRAGMENTATION STUDIES OF 158 A GeV Pb IONS USING CR39 NUCLEAR TRACK DETECTORS
}

\author{
H. Dekhissi ${ }^{1,2}$, G. Giacomelli ${ }^{1}$, M. Giorgini ${ }^{1}$, S. Manzoor ${ }^{1,3}$, L. Patrizii ${ }^{1}$, \\ V. Popa ${ }^{1,4}$, P. Serra ${ }^{1}$ and V. Togo ${ }^{1}$ \\ ${ }^{1}$ University of Bologna and INFN Sezione di Bologna, V. Berti Pichat 6/2, \\ Bologna I-40127, Italy \\ ${ }^{2}$ Faculty of Sciences, University Mohamed I, B.P. 㑩年 Oujda, Morocco and \\ ICTP, Trieste, Italy \\ ${ }^{3}$ Radiation Physics Div., PINSTECH, Islamabad, Pakistan and ICTP, \\ Trieste, Italy \\ ${ }^{4}$ Institute of Space Sciences, Bucharest R-76900, Romania
}

\begin{abstract}
Six stacks of CR39 ${ }^{1}$ nuclear track detectors with different targets were exposed to a lead beam of $158 \mathrm{~A} \mathrm{GeV}$ at the CERN-SPS, in order to study the fragmentation properties of ultra-relativistic lead nuclei. The exposures were performed at normal incidence with a mean density of about 2000 lead ions $/ \mathrm{cm}^{2}$ in the beam central area. The typical number of events in a stack was about $5.8 \times 10^{4}$ distributed in 8 spots. We present experimental results on lead fragmentation and charge pick-up cross sections.
\end{abstract}

\section{Introduction}

The study of the fragmentation properties of high energy atomic nuclei is of relevance for nuclear physics, cosmic ray physics and astrophysics. For instance, fragmentation cross sections are needed to evaluate the changes in composition undergone by cosmic ray particles in the collisions with the interstellar medium.

In this paper we present results on the fragmentation cross sections of 158 A $\mathrm{GeV}$ lead ions (charge $Z=82$ ); in particular the total charge changing

\footnotetext{
${ }^{1} \mathrm{CR} 39,\left(\mathrm{C}_{12} \mathrm{H}_{18} \mathrm{O}_{7}\right)_{n}$, is a Registered Trade Mark of PPG Industries Inc.
} 
cross sections, the partial fragmentation cross sections for $\Delta Z=-1,-2,-3$, and the $\Delta Z=+1$ pick-up cross sections were measured. The measurements were performed on different targets: $\mathrm{CR} 39, \mathrm{~Pb}, \mathrm{Cu}, \mathrm{Al}, \mathrm{C}$ and $\left(\mathrm{CH}_{2}\right)_{n}$ and composite targets. We have also evaluated the cross sections in Hydrogen and Oxygen.

The CR39 detectors were manufactured by the Intercast Europe Co. of Parma (Italy) and are similar to those used in a large area experimental search for magnetic monopoles at the Gran Sasso Laboratory (the MACRO experiment) [1].

Previous results concerning the fragmentation of $158 \mathrm{~A} \mathrm{GeV}$ lead ions, relative to a different exposure and with lower statistics were published in ref. [2].

\section{Experimental procedure}

Six stacks made of CR39 nuclear track detectors and different targets were exposed in November 1996 at the CERN-SPS to a beam of $158 \mathrm{~A} \mathrm{GeV}$ $\mathrm{Pb}^{82+}$ ions. The exposures were performed at normal incidence. The total number of lead ions in a stack was about $5.8 \times 10^{4}$ distributed in 8 spots. The average beam particle density was about 400 ions $/ \mathrm{cm}^{2}$; the central density in the spots was around 2000 ions $/ \mathrm{cm}^{2}$. The percentage of impurities in the beam was measured to be less than $1 \%$. The present analysis refers to data measured in a single spot for each target.

Each stack had the following composition (Fig. 1): (i) a $1.4 \mathrm{~mm}$ thick CR39 foil (which we refer to in the following as plate "A"); (ii) $7 \mathrm{~mm}$ of CR39 made up of layers of about $0.6 \mathrm{~mm}$ thickness; (iii) a target, typically $10 \mathrm{~mm}$ thick; (iv) about $15 \mathrm{~mm}$ of $0.6 \mathrm{~mm}$ CR39 layers; ( $v$ ) a $1.4 \mathrm{~mm}$ CR39 plate labelled " $\mathrm{B}$ ". The targets consisted of $\mathrm{Pb}, \mathrm{Cu}, \mathrm{Al}, \mathrm{C},\left(\mathrm{CH}_{2}\right)_{n}$ and CR39 sheets. In the present analysis only the CR39 plates "A" and "B" of Fig. 1 were measured; the others will be used for further analysis to improve the tracking reconstruction and the charge resolution.

After exposure, the CR39 detectors were etched for $268 \mathrm{~h}$ in a $6 \mathrm{~N} \mathrm{NaOH}$ water solution at a temperature of $45^{\circ} \mathrm{C}$. When an ion crosses a nuclear track detector foil, it produces damages at the level of molecular bonds forming the so called latent track. During the chemical etching of the detector, if the etching velocity along the latent track, $v_{T}$, is larger than that in the bulk material, $v_{B}$, etch-pit cones are formed on both sides of the foil. The 
base area and the height of the cones are functions of the Restricted Energy Loss (REL) of the incident ion [3-5]. After etching, the etch-pit cone base diameter of a track produced by a $\mathrm{Pb}$ ion is around $75 \mu \mathrm{m}$. The calibration of our CR39, using the present etching conditions, was discussed in detail in refs. [3, 4].

The base area of each etch-pit cone, its eccentricity and central brightness were measured on both sides of the "A" and "B" plates with an automatic image analyzer, the Elbek system [6]. For relativistic ions, using only two independent measurements, this technique has an acceptable charge resolution up to $Z \sim 75$; for $Z \geq 75$ the fragment tracks cannot be distinguished from the lead tracks [3]. For each beam spot, the number of tracks retained after a tracking procedure is about 5000 before the target; after the target the number of tracks with $Z \geq 75$ are about 2000-2600.

In order to determine the percentage of different ion charges in the range $75 \leq Z \leq 83$, we performed cone height measurements on a single face before and after the targets, inside the same spot measured by the Elbek system.

The height measurements were made with a microscope with a magnification of $(20)_{o b} \times(10)_{o c}$. About 6500 tracks (in two stacks) before the target, and about 3300 tracks after the target (for each stack), with base diameters larger than $75 \mu \mathrm{m}$, were manually measured. The charge resolution obtained from this measurement technique is about $0.2 e$ [3]. Fig. 2 shows the cone height distribution measured in plate " $\mathrm{B}$ " of the stack containing the lead target.

The percentages of non-lead tracks in the sample defined by diameter $\geq 75 \mu \mathrm{m}$ are about $1 \%$ before and $18 \% \div 35 \%$ after the different targets.

\section{Fragmentation of lead beam ions in the CR39 target}

The CR39 target was used to determine the cross section on the CR39 itself and to extract the cross sections for the other targets. It was used also with the $\left(\mathrm{CH}_{2}\right)_{n}$ and $\mathrm{C}$ targets to compute the cross sections in Hydrogen and Oxygen.

The total and partial fragmentation cross sections were calculated using the following propagation formula, see refs. $[7,8]$ 


$$
N_{i}(x)=N_{i}(0) e^{-x \sigma_{i}}+\sum_{j=1}^{i-1} K_{i j}\left(e^{-x \sigma_{i}}-e^{-x \sigma_{j}}\right)
$$

with

$$
K_{i j}=\frac{1}{\sigma_{j}-\sigma_{i}}\left(\sigma_{i j} N_{j}(0)-\sum_{l=j+1}^{i-1} \sigma_{i l} K_{l j}+\sum_{l=1}^{j-1} \sigma_{i j} K_{j l}\right)
$$

where $x=\rho t N_{A} / A, N_{A}$ is Avogadro's number, $\rho$ is the target density in $\mathrm{g} / \mathrm{cm}^{3}, A$ is the atomic mass, $t$ is the target thickness in $\mathrm{cm}, N_{i}(0)$ is the number of nuclei with charge $Z_{i}$ reaching the target $(i=1 \div 4$, corresponding to $Z=82 \div 79), N_{i}(x)$ is the number of ions of charge $Z_{i}$ after the target, $\sigma_{i}$ is the total fragmentation cross section of ions with charge $Z_{i}$, and $\sigma_{i j}$ is the partial fragmentation cross section for nuclei of charge $Z_{j}$ yielding a fragment of charge $Z_{i}$.

As an input for the computation we need the total charge changing cross sections $\sigma_{i}(i=1 \div 4)$ and the partial charge changing cross sections $\sigma_{i j}$. The total charge changing cross section for lead nuclei $\left(\sigma_{1}\right)$ was experimentally measured. For the fragments, the $\sigma_{i}$ were evaluated by scaling $\sigma_{1}$ according to the following equation

$$
\sigma_{i}=\sigma_{n i} / \sigma_{n 1}
$$

where, see ref. [9],

$$
\sigma_{n i}=10 \pi r_{0}^{2}\left[A_{i}^{1 / 3}+A_{t}^{1 / 3}-b\left(A_{i}^{-1 / 3}+A_{t}^{-1 / 3}\right)\right]^{2}
$$

In ref. [9], Eq. (4) was used to fit the data at $1 \div 2 \mathrm{~A} \mathrm{GeV}$ for projectiles and targets of atomic mass $A_{i} \geq 12$ and $A_{t} \geq 12$, respectively; for the parameters $r_{0}$ and $b$, ref. [10] gives the numerical values: $r_{0}=1.39 \mathrm{fm}$ and $b=1.65$.

The partial fragmentation cross sections $\sigma_{i 1}$ were experimentally measured; the other partial fragmentation cross sections $\sigma_{i j}$ were evaluated using a constant fragmentation probability for the same $\Delta Z$.

The measured partial charge changing cross sections in CR39 are given in Table 1, where the quoted errors are the sums in quadrature of statistical and systematic uncertainties. Note that the total charge changing cross section of $158 \mathrm{~A} \mathrm{GeV}$ lead nuclei in CR39 is $\sigma_{C R 39}=(2590 \pm 190) \mathrm{mb}$. The estimated systematic uncertainties are around $5 \%$. They are due to several sources; the 
main contribution arises from the relatively poor charge resolution at $Z \sim 74$ for measurements performed with the automatic Elbek system.

\section{Fragmentation of incident lead ions in various targets}

The total and partial fragmentation cross sections of lead ions in various targets were computed using the propagation formula (1), and are listed in Table 1. The results described in the previous Section were used to take into account the fragmentation in the CR39 foils.

The measured cross sections are the sum of the nuclear $\left(\sigma_{n}\right)$ and electromagnetic $\left(\sigma_{e m}\right)$ cross sections. The difference between the total cross section $\left(\sigma_{\text {tot }}\right)$, experimentally measured, and the nuclear cross section $\left(\sigma_{n}\right)$, computed using Eq. (4), is the electromagnetic contribution $\sigma_{e m}$, which can be described by the exponential part of formula (5).

The measured values for the total cross sections are listed in Table 1 and shown in Fig. 3 versus the target atomic mass.

The fit of our data for $A_{t} \leq 23$ to Eq. (4) gives $r_{0}=(1.32 \pm 0.05) \mathrm{fm}$, $b=(1.03 \pm 0.40)$ and $\chi^{2} / D . o . F .=1.3$ (in this $A_{t}$ region, the electromagnetic contribution to the total cross section is small). The dashed line in Fig. 3 has been computed with Eq. (4) using the parameters obtained for the fit for $A_{t} \leq 23$.

In order to include a Coulomb term, we used the following equation, see ref. [11],

$$
\sigma_{\text {tot }}=10 \pi r_{0}^{2}\left[A_{p}^{1 / 3}+A_{t}^{1 / 3}-b\left(A_{p}^{-1 / 3}+A_{t}^{-1 / 3}\right)\right]^{2}+\alpha Z^{\delta} .
$$

Assuming $r_{0}=1.32 \mathrm{fm}$ and $b=1.03$ from our previous fit, and $\alpha=0.6 \mathrm{mb}$ from ref. [11], we fitted our data to Eq. (5) obtaining $\delta=(2.05 \pm 0.05)$ with $\chi^{2} /$ D.o.F. $=1.16$. The result of the fit is the solid line in Fig. 3.

The measured partial cross sections for $\Delta Z=-1,-2,-3$ are listed in Table 1 for different targets.

The cross sections on Hydrogen and Oxygen targets have been determined using the measured cross sections in CR39, $\left(\mathrm{CH}_{2}\right)_{n}$ and $\mathrm{C}$.

\section{Charge pick-up cross section}

The charge pick-up reaction, in which the projectile nucleus increases its charge number by one unit, was also studied. The pick-up cross sections for 


\begin{tabular}{|c|c|c|c|c|c|c|}
\hline Target & $A_{t}$ & $\sigma_{\text {tot }}$ & $\sigma_{(\Delta Z=-1)}$ & $\sigma_{(\Delta Z=-2)}$ & $\sigma_{(\Delta Z=-3)}$ & $\sigma_{(\Delta Z=1)}$ \\
\hline $\mathrm{H}$ & 1 & $1940 \pm 234$ & $765 \pm 91$ & $117 \pm 36$ & $105 \pm 38$ & $15 \pm 8$ \\
\hline $\mathrm{CH}_{2}$ & 4.67 & $2421 \pm 181$ & $522 \pm 61$ & $92 \pm 23$ & $98 \pm 24$ & $13 \pm 5$ \\
\hline$\left(\mathrm{CH}_{2}\right)_{n}+\mathrm{CR} 39$ & 6.45 & $2576 \pm 188$ & $335 \pm 53$ & $112 \pm 17$ & $95 \pm 17$ & $11 \pm 4$ \\
\hline $\mathrm{CR} 39$ & 7.4 & $2590 \pm 190$ & $221 \pm 29$ & $117 \pm 31$ & $90 \pm 25$ & $7 \pm 4$ \\
\hline $\mathrm{C}+\mathrm{CR} 39$ & 8.96 & $2900 \pm 201$ & $164 \pm 35$ & $98 \pm 18$ & $89 \pm 18$ & $10 \pm 4$ \\
\hline $\mathrm{Al+CR39}$ & 11.5 & $3228 \pm 229$ & $243 \pm 51$ & $156 \pm 27$ & $103 \pm 20$ & $17 \pm 6$ \\
\hline $\mathrm{C}$ & 12 & $3382 \pm 220$ & $36 \pm 16$ & $42 \pm 23$ & $83 \pm 26$ & $9 \pm 5$ \\
\hline $\mathrm{O}$ & 16 & $2904 \pm 959$ & - & - & - & - \\
\hline $\mathrm{Cu}+\mathrm{CR} 39$ & 22.9 & $3610 \pm 243$ & $322 \pm 56$ & $168 \pm 29$ & $74 \pm 14$ & $18 \pm 7$ \\
\hline $\mathrm{Al}$ & 27 & $4651 \pm 349$ & $246 \pm 68$ & $243 \pm 57$ & $122 \pm 40$ & $34 \pm 12$ \\
\hline $\mathrm{Pb}+\mathrm{CR} 39$ & 32.5 & $3832 \pm 263$ & $690 \pm 98$ & $282 \pm 36$ & $133 \pm 20$ & $21 \pm 7$ \\
\hline $\mathrm{Cu}$ & 63.5 & $6108 \pm 384$ & $574 \pm 78$ & $284 \pm 59$ & $27 \pm 23$ & $27 \pm 12$ \\
\hline $\mathrm{Pb}$ & 207 & $11982 \pm 762$ & $3920 \pm 413$ & $1403 \pm 176$ & $417 \pm 81$ & $74 \pm 27$ \\
\hline
\end{tabular}

Table 1: The measured total, partial break-up and pick-up charge changing cross sections for lead ions of $158 \mathrm{~A} \mathrm{GeV}$ on various targets. All values are in mb; quoted uncertainties are the sums in quadrature of statistical and systematic uncertainties.

the different targets were determined using the equation (see ref. [12]),

$$
\sigma_{p 1}=\frac{1}{x} \frac{N_{p}(x)}{N_{1}(x)}
$$

where $N_{p}$ is the number of nuclei with $Z=83$ produced inside the target, $N_{1}(x)$ is the number of unfragmented beam nuclei and $x$ has the same meaning as in Eq. (1). $\sigma_{p 1}$ was directly measured for the CR39 target. For the other targets we computed $N_{p}(x)$ as

$$
N_{p}(x)=N_{p}^{\prime}(x)-N_{p}^{\prime}(0) \cdot e^{-x \sigma_{p}}
$$

where $N_{p}^{\prime}(x)$ and $N_{p}^{\prime}(0)$ are the computed numbers of $Z=83$ nuclei immediately after and before the target and $\sigma_{p}$ is their estimated fragmentation cross section.

\section{Conclusions}

Using stacks of CR39 nuclear track detectors, we measured the total, partial break-up and pick-up charge changing cross sections for lead nuclei 
of $158 \mathrm{~A} \mathrm{GeV}$ in different targets. The experimental total charge changing cross sections can be described by a semiempirical formula which takes into account nuclear and electromagnetic processes.

We have tried to compare our measurements with the data available in the literature relative to lower beam energies and different beam ions [1115]. The comparison is difficult; nevertheless the data indicate a similar $A_{t}$ dependence of the cross sections.

\section{Acknowledgements}

We would like to thank the CERN SPS staff for the good performance of the $\mathrm{Pb}$ beam and for the exposures. We acknowledge the contribution of our technical staff. H. D. and S. M. thank the ICTP/Trieste Programme on Training and Research in Italian Laboratories for providing fellowships. V. P. thanks INFN for hospitality and financial support. 


\section{References}

[1] MACRO Collaboration, S. Ahlen et al., Nucl. Instr. Meth. A 324 (1993) 337.

[2] G. Giacomelli et al., 25 $5^{\text {th }}$ International Cosmic Ray Conference, Durban, Vol. 6 (1997) 17.

[3] G. Giacomelli et al., Nucl. Instr. Meth. A 411 (1998) 41.

[4] S. Cecchini et al., Nuovo Cim. A 109 (1996) 1119.

[5] R. Fleischer, P. B. Price and R. M. Walker, Nuclear Tracks in Solids, University of California Press (1975).

[6] A. Noll et al., Nucl. Tracks Radiat. Meas. 15 (1988) 265.

[7] C. Brechtmann and W. Heinrich, Nucl. Instr. Meth. B 29 (1988) 675.

[8] S. Cecchini et al., Astrop. Phys. 1 (1993) 369.

[9] H. H. Heckman et al., Phys. Rev. C 17 (1978) 1735.

[10] G. D. Westfall et al., Phys. Rev. C 19 (1979) 1309.

[11] Y. He and P. B. Price, Z. Phys. A 348 (1994) 105.

[12] D. P. Bhattacharyya et al., Nuovo. Cim. 16 C (1993) 249.

[13] D. Sampsonidis et al., Phys. Rev. C51 (1995) 3304.

[14] P. J. Lindstrom et al., Bull. Am. Phys. Soc. 17 (1970) 54.

[15] S. E. Hirzebruch et al., Phys. Rev. C51 (1995) 2085. 


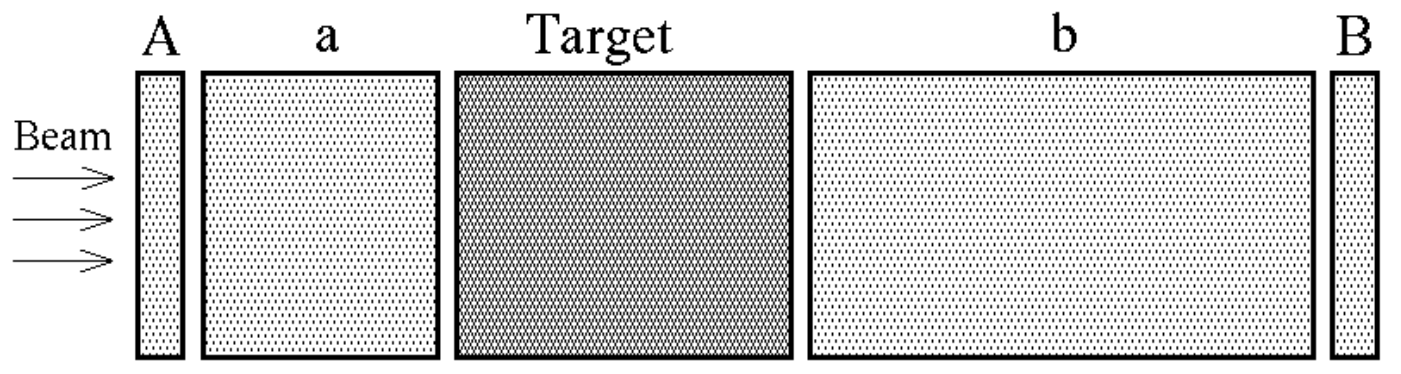

Figure 1: Sketch of a stack used for the fragmentation studies. "A" and "B" are $1.4 \mathrm{~mm}$ thick CR39 sheets, "a", "b" are stacks about $1 \mathrm{~cm}$ thick of layers of 0.6 $\mathrm{mm}$ thick CR39; the target is typically $10 \mathrm{~mm}$ thick. 


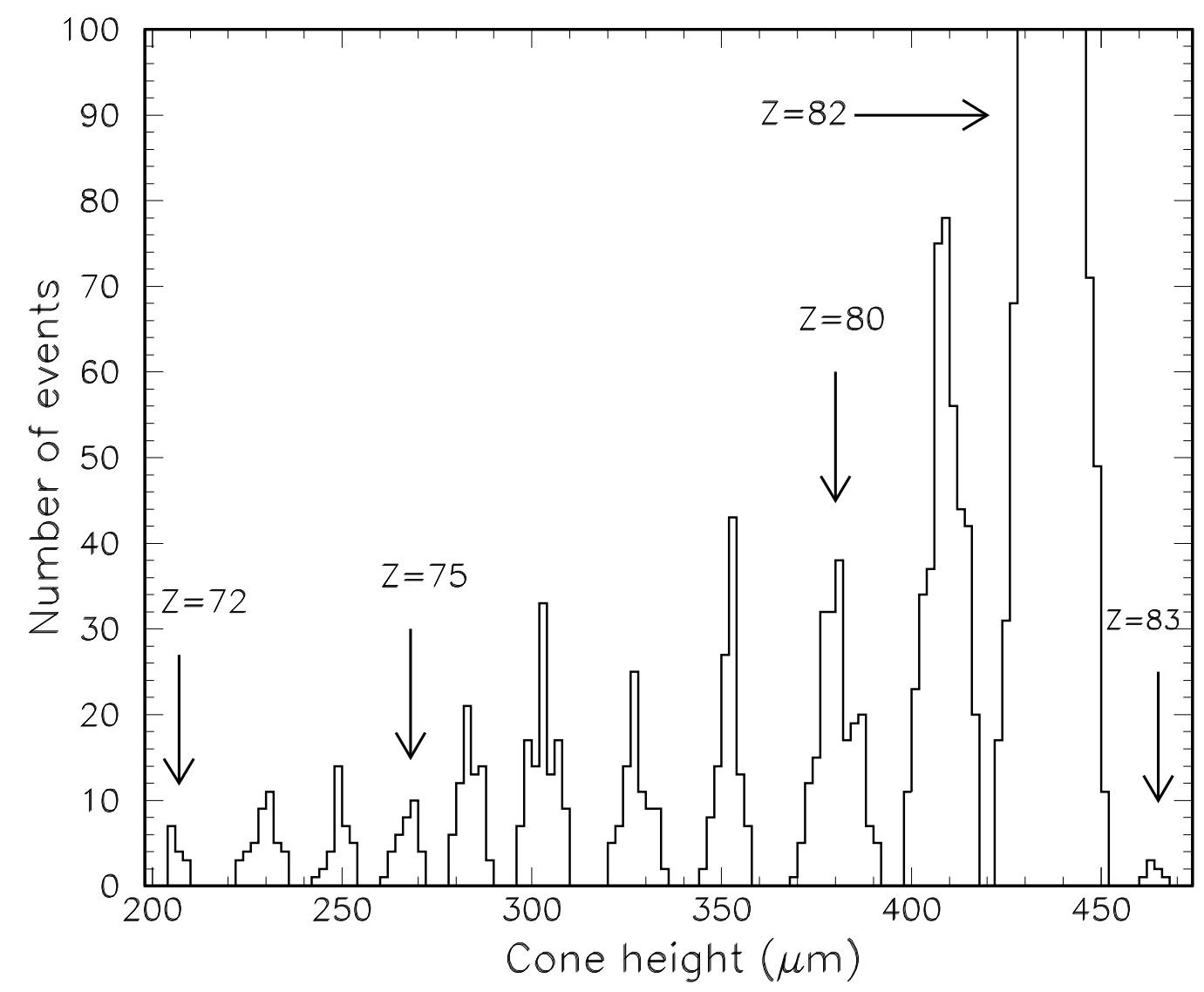

Figure 2: Distribution of etched cone heights for the "B" CR39 sheet located after the lead target. 


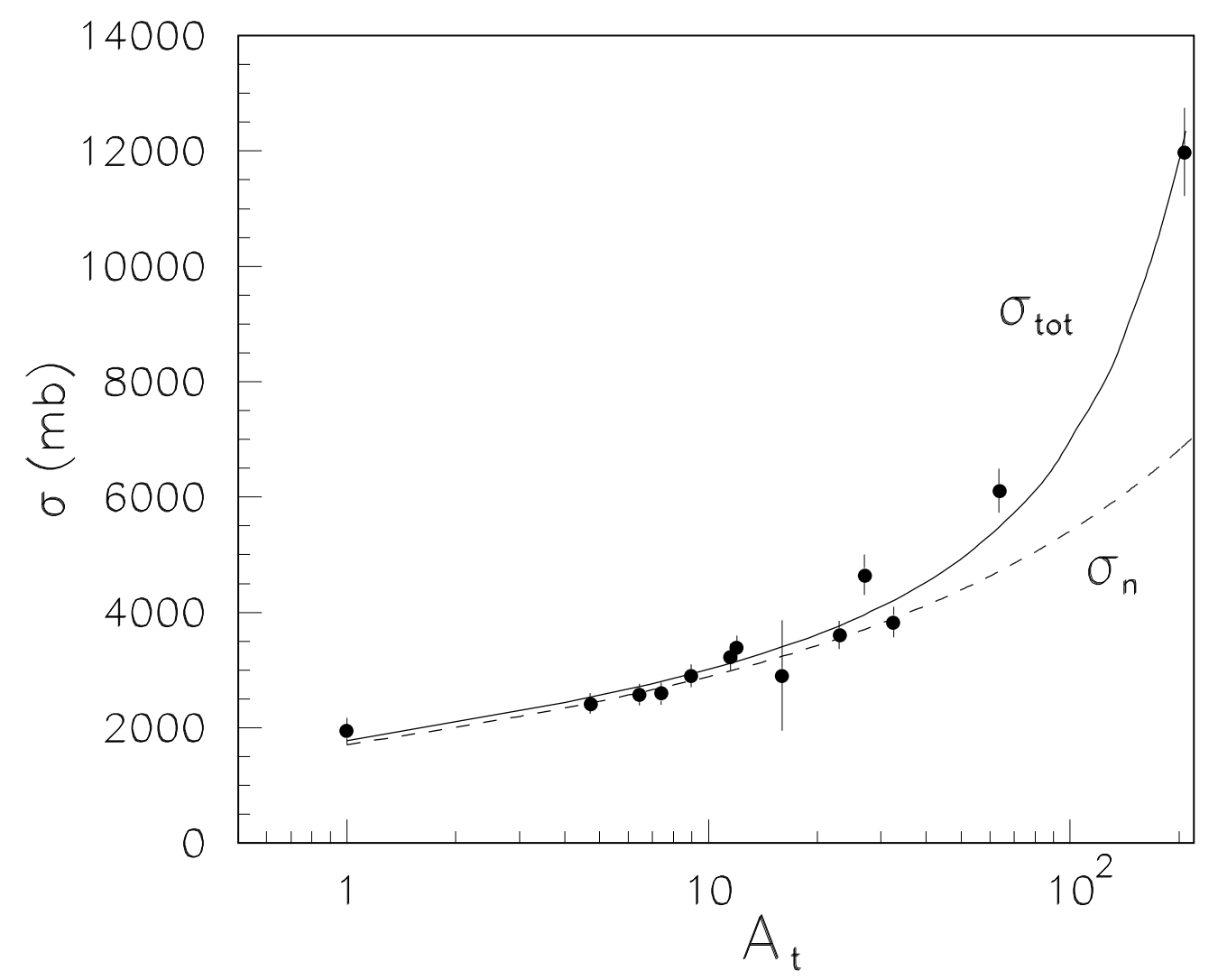

Figure 3: Measured total fragmentation charge changing cross sections for 158 A GeV Pb projectiles in various targets (black points). The solid line represents the fit of our data to formula (5); the dashed line is the nuclear cross section contribution (see text). 